\title{
Hartogs theorem for forms: solvability of Cauchy-Riemann operator at critical degree
}

\author{
Chin-Huei Chang And Hsuan-Pei LeE
}

\begin{abstract}
The Hartogs Theorem for holomorphic functions is generalized in two settings: a CR version (Theorem 1.2) and a corresponding theorem based on it for $C^{k} \bar{\partial}$-closed forms at the critical degree, $0 \leq k \leq \infty$ (Theorem 1.1). Part of Frenkel's lemma in $C^{k}$ category is also proved.
\end{abstract}

Mathematics Subject Classification (2000): 32A26 (primary); 32W10 (secondary).

\section{Introduction}

Let $P_{N}$ denote the unit polydisc in $\mathbb{C}^{N}, N \geq 1$. In $\mathbb{C}^{m+1}, m \geq 1$, set

$$
\omega=P_{m} \times\left\{z_{m+1} \in \mathbb{C}\left|\frac{1}{2}<\right| z_{m+1} \mid<1\right\}, \quad m \geq 1 .
$$

The classical Hartogs theorem (see [9, p. 55]) states: suppose, for a given holomorphic function $f$ on $\omega$, there is an open set $U \subset P_{m}$ such that $f$ has a holomorphic extension to $U \times\left\{z_{m+1} \in \mathbb{C}|| z_{m+1}|<1|\right\}$, then $f$ can be extended holomorphically to $P_{m+1}$. This phenomenon in higher fiber dimension is suggested by Frenkel's lemma (see [13, p. 15]).

Let $n$ always be an integer bigger than 1 . For $z \in \mathbb{C}^{m+n}$ we write $z=\left(z^{\prime}, z^{\prime \prime}\right)$ with $z^{\prime}=\left(z_{1}, \ldots, z_{m}\right)$ and $z^{\prime \prime}=\left(z_{m+1}, \ldots, z_{m+n}\right)$. Set

$$
\Omega=P_{m} \times\left(P_{n} \backslash \frac{1}{2} P_{n}\right)
$$

where $\frac{1}{2} P_{n}=\left\{z^{\prime \prime} \in \mathbb{C}^{n} \mid \quad 2 z^{\prime \prime} \in P_{n}\right\}$. The first part of Frenkel's lemma says: the Cauchy-Riemann equation

$$
\bar{\partial} u=f, \quad f \in C_{(0, q)}^{\infty}(\Omega), \quad 1 \leq q \leq m+n, \quad \bar{\partial} f=0
$$

Received July 29, 2005; accepted in revised form January 5, 2006. 
always has a solution $u \in C_{(0, q-1)}^{\infty}(\Omega)$ except $q=n-1$. From now on a $(0, n-1)$ form will be called of the "critical degree".

Note that in Hartogs theorem the open set $U$ can be replaced by a subset $A$ of $P_{m}$ such that $A$ is not contained in a subvariety of codimension one in $P_{m}$ (see [12, p. 16]). The following is our first theorem.

Theorem 1.1. With $\Omega$, A as above. Let $f$ be a $C^{k} \bar{\partial}$-closed $(0, n-1)$ form on $\Omega$, $0 \leq k \leq \infty$. For $z^{\prime} \in P_{m}$, let $\gamma_{z^{\prime}}=\Omega \cap\left(\left\{z^{\prime}\right\} \times \mathbb{C}^{n}\right)$ be the fiber over $z^{\prime}$ in $\Omega$. For every $z^{\prime} \in A$, suppose the Cauchy-Riemann equation on $\gamma_{z^{\prime}}$,

$$
\bar{\partial}_{\gamma_{z^{\prime}}} v=f
$$

is solvable. Then the Cauchy-Riemann equation on $\Omega$

$$
\bar{\partial} v=f
$$

is solvable with $v \in C^{k}(\Omega)$.

We explain the notation for the (tangential) Cauchy-Riemann operator used in this paper. In general, if there is no ambiguity, it is denoted by $\bar{\partial}$; if the ground space $X$ is specified, we use $\bar{\partial}_{X}$ to denote the Cauchy-Riemann (or tangential CauchyRiemann) operator on $X$. Also in integral representations, we usually use $\zeta$ for the dummy variable and $z$ for the resulting variable. In this case, the notation $\bar{\partial}_{\zeta}$ (respectively, $\bar{\partial}_{z}$ ) denotes the (tangential) Cauchy-Riemann operator with respect to $\zeta$ (respectively, $z$ ) variable.

The proof of Theorem 1.1 depends on Theorem 1.2 which is the CR version of Theorem 1.1. Let $\rho$ be a $C^{k}(k \geq 3)$ real valued function in $\mathbb{C}^{m+n}$ which is strictly plurisubharmonic in a neighborhood of $\{\rho \leq 0\}$. Let $\sigma$ be a $C^{k}$ real valued function in $\mathbb{C}^{m}$, strictly plurisubharmonic in a neighborhood of $\{\sigma \leq 0\}$. We also assume that $\{\sigma<0\}$ is connected and relatively compact in $\mathbb{C}^{m}$. Set

$$
M=\{\rho=0\} \cap\left(\{\sigma<0\} \times \mathbb{C}^{n}\right) .
$$

Assume that $d \rho$ (respectively, $d \sigma$ ) does not vanish on $\{\rho=0\}$ (respectively, $\{\sigma=$ $0\})$ and $d \rho \wedge d \sigma \neq 0$ on $\partial M$. Let $f$ be a $(0, q)$ form on $M$ such that $\bar{\partial}_{M} f=0$ in distribution sense. It is proved in [1] that if $f \in L_{(0, q)}^{p}(M)$ (i.e. $f$ is a $(0, q)$ form with coefficients in $\left.L^{p}\right), 1 \leq p \leq \infty$, then

$$
\bar{\partial}_{M} u=f
$$

is solvable on $M$ with $u \in L_{(0, q-1)}^{p}(M)$ whenever $1 \leq q<n-1$. In the case $q=n-1$, the above equation is solvable with $f \in C_{(0, q)}^{0}(\bar{M})$ (i.e. coefficients of $f$ are continuous on $\bar{M}$,) if and only if $f$ satisfies the moment condition. Our main result is the following: 
Theorem 1.2. Let $M$ be as above and $A$ be a subset of $\{\sigma<0\}$ not contained in any subvariety of codimension one in $\{\sigma<0\}$. Let $f$ be a $\bar{\partial}$-closed $(0, n-1)$ form with $f \in C_{(0, n-1)}^{0}(\bar{M}) \cap C^{k^{\prime}}(M), 0 \leq k^{\prime} \leq k-3$. The equation (*) is solvable with $u \in C_{(0, n-2)}^{k^{\prime}}(M)$ if and only if all the holomorphic moments of $f$ on $\Gamma_{z^{\prime}}, z^{\prime} \in A$ vanish, in other words, for every $z^{\prime} \in A$

$$
\int_{\Gamma_{z^{\prime}}} h\left(z^{\prime}, \zeta^{\prime \prime}\right) f\left(z^{\prime}, \zeta^{\prime \prime}\right) d \zeta^{\prime \prime}=0
$$

for every function $h$ holomorphic near $\Gamma_{z^{\prime}}$, where $\Gamma_{z^{\prime}}=M \cap\left(\left\{z^{\prime}\right\} \times \mathbb{C}^{n}\right)$ is the fiber over $z^{\prime}$ in $M$.

\section{Remark 1.3.}

(a) When $\Gamma_{z^{\prime}}$ is strictly pseudoconvex in $\left\{z^{\prime}\right\} \times \mathbb{C}^{n}$, it is well-known that the solvability of the tangential Cauchy-Riemann equation $\bar{\partial}_{\Gamma_{z^{\prime}}} u=f$ with $f$ of top degree is equivalent to the vanishing of all holomorphic moments of $f$ on $\Gamma_{z^{\prime}}$. So if $\Gamma_{z^{\prime}}$ is strictly pseudoconvex in $\left\{z^{\prime}\right\} \times \mathbb{C}^{n}$ for every $z^{\prime} \in A$, the statement of Theorem 1.2 can be phrased as:

The equation (*) is solvable with $u \in C^{0}(M)$ if and only if $\bar{\partial}_{\Gamma_{z^{\prime}}} v=f$ is solvable for every $z^{\prime} \in A$.

(b) The CR function version of Hartogs theorem was proved in [2] where the condition for $A$ is stronger but no convexity condition or boundary regularity is required for $M$.

We recall the representaion formula for $\bar{\partial}_{b}$-closed form $f$ on $M$ (cf. [1]):

$$
\begin{aligned}
(-1)^{q} f(z)= & \bar{\partial}\left\{\int_{M} f(\zeta) \wedge \Omega_{q-1}\left(\mathfrak{r}, \mathfrak{r}^{*}\right)(\zeta, z)+(-1)^{q} \int_{\partial M} f(\zeta) \wedge \Omega\left(\mathfrak{r}, \mathfrak{r}^{*}, \mathfrak{s}\right)(\zeta, z)\right\} \\
& +\int_{\partial M} f(\zeta) \wedge \Omega_{q}\left(\mathfrak{r}^{*}, \mathfrak{s}\right)(\zeta, z), \quad z \in M
\end{aligned}
$$

where $\Omega\left(\mathfrak{r}, \mathfrak{r}^{*}\right), \Omega\left(\mathfrak{r}, \mathfrak{r}^{*}, \mathfrak{s}\right), \Omega\left(\mathfrak{r}^{*}, \mathfrak{s}\right)$ are defined in Section 2. It is clear from this representation that the obstruction to the solvability of $(*)$ is the last integral which is null when $q<n-1$ by type consideration. We therefore in Section 2 define for $f \in C_{(0, n-1)}^{0}(\bar{M}), \bar{\partial}_{M} f=0$ in distribution sense, the following transform:

$$
T f(z)=(-1)^{n-1} \int_{\partial M} f(\zeta) \wedge \Omega\left(\mathfrak{r}^{*}, \mathfrak{s}\right)(\zeta, z),
$$

which may be taken as the global moment of $f$ (cf. $\left(\left(3.1^{\prime}\right)\right)$ ). Section 3 consists of the properties of the operator $T$ needed in this paper. For $f$ in the domain of $T$ 
we show that $T f$ is defined in a set containing $M$ and is $\bar{\partial}$-closed there, so (2.7) becomes a "jump formula" for $f$ (see Remark 3.3(a) for more details). Next, we show that $T$ can be defined locally over the base set $\{\sigma<0\}$. Finally, the operator $T$ is proved to be just defined fiberwise over every point $z^{\prime} \in\{\sigma<0\}$. This enables us to define $T$ on $M$ with arbitrary base set $B$ in $\mathbb{C}^{m}$. In section 4 we define the moment operator $\mathcal{M}_{h}$ for $T f$ (or $f$ ) with respect to a holomorphic function $h$. It turns out that $\mathcal{M}_{h}(f)$ is a holomorphic function in the base set. Using this property we prove a more general Theorem 4.4 which implies Theorem 1.2 immediately. Section 5 contains the proof of Theorem 1.1. The interesting thing here is a procedure which improves the method in [11] to produce a $C^{k}$ solution for $0 \leq k \leq \infty$. The results of this paper hold for $(p, n-1)$ forms, $0 \leq p \leq n$. For simplicity, we only deal with the case $p=0$.

Finally, closely related to the topics in this paper, there is another Hartogs theorem (see [9, p. 56, 63]) whose higher dimensional analogue is written in a forthcoming paper.

ACKNOWLEDGEMENTS. We thank the referee for comments that helped to improve the presentation of this paper.

\section{Preliminaries}

We write $\zeta \in \mathbb{C}^{m+n}$ as $\left(\zeta^{\prime}, \zeta^{\prime \prime}\right)$ where $\zeta^{\prime} \in \mathbb{C}^{m}$ and $\zeta^{\prime \prime} \in \mathbb{C}^{n}$. Similarly, for differential forms we write $d f=\left(d^{\prime} f, d^{\prime \prime} f\right)$, and $\partial f=\left(\partial^{\prime} f, \partial^{\prime \prime} f\right)$, where $d^{\prime} \cdot, d^{\prime \prime}$. denote respectively the differentials with repect to the first $2 m$ variables and those with respect to the last $2 n$ variables; likewise for $\partial^{\prime} \cdot\left(\bar{\partial}^{\prime} \cdot\right)$ and $\partial^{\prime \prime} \cdot\left(\bar{\partial}^{\prime \prime} \cdot\right)$. Also we use $d \zeta=d \zeta_{1} \wedge \ldots \wedge d \zeta_{m+n}, d \zeta^{\prime}=d \zeta_{1} \wedge \ldots \wedge d \zeta_{m}$ and $d \zeta^{\prime \prime}=d \zeta_{m+1} \wedge \ldots \wedge d \zeta_{m+n}$ similarly for $d \bar{\zeta}, d \bar{\zeta}^{\prime}, d \bar{\zeta}^{\prime \prime}$, etc..

The following notations and exterior calculus developed by Harvey and Polking [5] will be used in construting kernels needed in this paper:

Let $E^{1}, \ldots, E^{\alpha}$ (which are called sections) be a collection of $N$-tuples of $C^{2}$ functions in $(\zeta, z) \in \mathbb{C}^{N} \times \mathbb{C}^{N}$. Following Harvey-Polking [5] we use

$$
\begin{aligned}
\Omega\left(E^{1}, \ldots, E^{\alpha}\right)= & \frac{\left\langle E^{1}, \vec{d} \zeta\right\rangle}{\left\langle E^{1}, \zeta-z\right\rangle} \wedge \cdots \wedge \frac{\left\langle E^{\alpha}, \vec{d} \zeta\right\rangle}{\left\langle E^{\alpha}, \zeta-z\right\rangle} \\
& \wedge \sum_{\lambda_{1}+\ldots+\lambda_{\alpha}=N-\alpha}\left(\frac{\left\langle\bar{\partial}_{\zeta, z} E^{1}, \vec{d} \zeta\right\rangle}{\left\langle E^{1}, \zeta-z\right\rangle}\right)^{\lambda_{1}} \wedge \cdots \wedge\left(\frac{\left\langle\bar{\partial}_{\zeta, z} E^{\alpha}, \vec{d} \zeta\right\rangle}{\left\langle E^{\alpha}, \zeta-z\right\rangle}\right)^{\lambda_{\alpha}}
\end{aligned}
$$

where $\langle x, y\rangle=\sum x_{i} y_{i}$ for vectors $x, y$ in $\mathbb{C}^{N}$ and $\vec{d} \zeta$ here is understood to be the $N$-vector $\left(d \zeta_{1}, \ldots, d \zeta_{N}\right)$. Then $\Omega$ is $C^{1}$ away from the singular set $Z=\bigcup_{1}^{\alpha}\left\{(\zeta, z) \mid\left\langle E^{j}, \zeta-z\right\rangle=0\right\}$. We can rewrite $\Omega$ as $\Omega\left(E^{1}, \ldots, E^{\alpha}\right)=$ $\sum_{0}^{N-1} \Omega_{q}\left(E^{1}, \ldots, E^{\alpha}\right)$, where $\Omega_{q}$ is the sum of components of $\Omega$ which are of 
degree $q$ in $d \bar{z}_{j}, j=1, \ldots, N$. Outside the singular set $Z$ we have the following identity:

$$
\bar{\partial}_{\zeta, z} \Omega\left(E^{1}, \ldots, E^{\alpha}\right)=\sum_{j=1}^{\alpha}(-1)^{j} \Omega\left(E^{1}, \ldots, \widehat{E^{j}}, \ldots, E^{\alpha}\right) .
$$

To construct the sections we use the results of Fornæss [3] which we briefly outline in the following and refer to [3] for details:

We first observe that for any strongly convex domain $G \subset \mathbb{C}^{N}$ with $C^{k}, k \geq 2$ boundary, there exist a $C^{k}$ function $\mu$ with positive real Hessian, a constant $c>0$ such that $G=\{\mu<0\}$, and $d \mu \neq 0$ in a neighborhood of $\partial G$. Furthermore, if we define

$$
\mathcal{H}(\xi, \eta)=\sum_{1}^{N} \frac{\partial \mu}{\partial \xi_{j}}(\xi)\left(\xi_{j}-\eta_{j}\right)
$$

then it satisfies

$$
\mathcal{H}(\xi, \eta) \geq \mu(\xi)-\mu(\eta)+c|\xi-\eta|^{2}
$$

for all $\xi, \eta$ in a small neighborhood of $\bar{G}$. The section $\left(\frac{\partial \mu}{\partial \xi_{1}}(\xi), \cdots, \frac{\partial \mu}{\partial \xi_{N}}(\xi)\right)$ will serve the purpose of this paper in case the given domain is strongly convex.

On the other hand, Fornæss proved in [3] that any strongly pseudoconvex domain $X \subset \mathbb{C}^{N}$ with $C^{k}, k \geq 2$ boundary, admits an embedding into a bounded strongly convex domain $Y \subset \mathbb{C}^{N^{\prime}}$ with $C^{k}$ boundary for some $N^{\prime}$, such that the boundary of $X$ is mapped into the boundary of $Y$, and the map is 1-1 holomophic in a neighborhood of $\bar{X}$ (cf. [3, Theorem 9] for explicit statements).

Now for any strongly pseudoconvex domain $X \subset \mathbb{C}^{N}$ with $C^{k}, k \geq 2$ boundary, the above oberservation and Fornæss' embedding theroem together imply the existence of $\mathcal{H}$ and the section for $Y \subset \mathbb{C}^{N^{\prime}}$. Their pull-backs to $\mathbb{C}^{N}$ then give the following resuts (cf. [3, Theorem 16]):

There exist a $C^{k}$ function $v$ which is strictly plurisubharmonic in a neighborhood of $\bar{X}$ with $X=\{v<0\}$, a constant $\epsilon>0$ and a function $H(\xi, \eta) \in$ $C^{k-1}\left(X_{\epsilon} \times X_{\epsilon}\right)$, where $X_{\epsilon}=\left\{\eta \in \mathbb{C}^{N}, v(\eta)<\epsilon\right\}$ satisfying

$$
H(\xi, \cdot) \text { is holomorphic in } X_{\epsilon},
$$

$\exists n_{j}(\xi, \eta) \in C^{k-1}\left(X_{\epsilon} \times X_{\epsilon}\right), j=1, \ldots, N$, holomorphic in $\eta$, such that

$$
\begin{gathered}
H(\xi, \eta)=\sum_{1}^{N} n_{j}(\xi, \eta)\left(\xi_{j}-\eta_{j}\right), \\
\exists c>0, \text { such that } \forall \eta \in \bar{X}, \xi \in \bar{X}
\end{gathered}
$$

$2 \operatorname{Re} H(\xi, \eta) \geq v(\xi)-v(\eta)+c|\xi-\eta|^{2}$,

$$
\left.d_{\xi} H(\xi, \eta)\right|_{\xi=\eta}=\partial \nu(\xi)
$$


Let $\rho, \sigma$ be as in Section 1. In view of the above discussion, for the strongly pseudoconvex domain $\{\rho<0\} \subset \mathbb{C}^{m+n}$ there exist $\mathfrak{r}_{j}$ 's which correspond to the $n_{j}$ 's in (2.4), and we define the section $\mathfrak{r}(\zeta, z)$ as $\left(\mathfrak{r}_{1}, \ldots, \mathfrak{r}_{m+n}\right)$. Similarly for the domain $\{\sigma<0\} \subset \mathbb{C}^{m}$ we define the section $\mathfrak{s}^{\prime}\left(\zeta^{\prime}, z^{\prime}\right)=\left(\mathfrak{s}_{1}, \ldots, \mathfrak{s}_{m}\right)$. We then use $\mathfrak{s}(\zeta, z)$ for the section $(\mathfrak{s}_{1}\left(\zeta^{\prime}, z^{\prime}\right), \ldots, \mathfrak{s}_{m}\left(\zeta^{\prime}, z^{\prime}\right), \underbrace{0, \cdots, 0}_{n})$. Let $\mathfrak{r}^{*}(\zeta, z)=$ $\left(\mathfrak{r}_{1}^{*}(\zeta, z), \ldots, \mathfrak{r}_{m+n}^{*}(\zeta, z)\right)$, where $\mathfrak{r}_{j}^{*}(\zeta, z)=-\mathfrak{r}_{j}(z, \zeta)$. Thus $\mathfrak{r}, \mathfrak{r}^{*}$ and $\mathfrak{s}$ are $C^{k-1}$ in a neighborhood of $\bar{M} \times \bar{M}$.

The kernels $\Omega\left(\mathfrak{r}, \mathfrak{r}^{*}\right), \Omega\left(\mathfrak{r}, \mathfrak{r}^{*}, \mathfrak{s}\right), \Omega\left(\mathfrak{r}^{*}, \mathfrak{s}\right)$ etc., are defined according to formula (2.1).

For $f \in C_{(0, q)}^{0}(\bar{M})$, satisfying $\bar{\partial}_{M} f=0$ in distribution sense on $M$ with $1 \leq q \leq n+m-1$, we recall the following basic representation formula from [1, p. 543]: for $z \in M$

$$
\begin{aligned}
(-1)^{q} f(z)= & \bar{\partial}\left\{\int_{M} f(\zeta) \wedge \Omega_{q-1}\left(\mathfrak{r}, \mathfrak{r}^{*}\right)(\zeta, z)+(-1)^{q} \int_{\partial M} f(\zeta) \wedge \Omega\left(\mathfrak{r}, \mathfrak{r}^{*}, \mathfrak{s}\right)(\zeta, z)\right\} \\
& +\int_{\partial M} f(\zeta) \wedge \Omega_{q}\left(\mathfrak{r}^{*}, \mathfrak{s}\right)(\zeta, z) .
\end{aligned}
$$

The last integral in (2.7) is null when $q<n-1$ by type consideration. For $f \in$ $C_{(0, n-1)}^{0}(\bar{M}), \bar{\partial}_{M} f=0$ in distribution sense, we define the following transform:

$$
T f(z)=(-1)^{n-1} \int_{\partial M} f(\zeta) \wedge \Omega\left(\mathfrak{r}^{*}, \mathfrak{s}\right)(\zeta, z) .
$$

Remark 2.1. A $(0, n-1)$ form $f$ defined in a subset of $\mathbb{C}^{m+n}$ can be decomposed as follows:

$$
f=\sum_{j=1}^{s} f_{j} \text { where } f_{j}=\sum_{\substack{|\alpha|+|\beta|=n-1 \\|\alpha|=j-1}} f_{j \alpha} d \bar{z}^{\prime \alpha} d \bar{z}^{\prime \prime \beta} \text { and } s=\min (m+1, n) .
$$

Moreover, when $f$ is $\bar{\partial}$-closed we have:

$$
\bar{\partial}_{z^{\prime \prime}} f_{1}=0, \bar{\partial}_{z^{\prime}} f_{j}=-\bar{\partial}_{z^{\prime \prime}} f_{j+1}, j=1, \ldots, s-1, \text { and } \bar{\partial}_{z^{\prime}} f_{s}=0 .
$$

The definition of $T$ immediately gives

$$
T f=T f_{1}=(T f)_{1} .
$$

\section{Properties of $\mathbf{T f}$}

In this section we always assume that $f \in C_{(0, n-1)}^{0}(\bar{M})$ and $\bar{\partial}_{M} f=0$ in distribution sense. 
Lemma 3.1. $T f=0$ if there exists $u \in C_{(0, n-2)}^{0}(\bar{M})$ such that $\bar{\partial}_{M} u=f$ on $M$.

Proof. Suppose there exists $u \in C_{(0, n-2)}^{0}(\bar{M})$ satisfying $\bar{\partial}_{M} u=f$. By the definition of $T f$, we have

$$
T f=(-1)^{n-1} \int_{\partial M} \bar{\partial}_{\zeta} u(\zeta) \wedge \Omega\left(\mathfrak{r}^{*}, \mathfrak{s}\right)(\zeta, z)=\int_{\partial M} u \wedge \bar{\partial}_{\zeta} \Omega\left(\mathfrak{r}^{*}, \mathfrak{s}\right)(\zeta, z)
$$

by Stokes' theorem. Invoking (2.2) the last integral becomes

$$
\int_{\partial M} u \wedge\left(\Omega\left(\mathfrak{r}^{*}\right)-\Omega(\mathfrak{s})-\bar{\partial}_{z} \Omega\left(\mathfrak{r}^{*}, \mathfrak{s}\right)\right)=0
$$

by type considerations. This proves the lemma.

Lemma 3.2. There is an open neighborhood $\mathcal{N}$ of $M$ in $\{\sigma<0\} \times \mathbb{C}^{n}$, depending on $\rho$ only, such that $T f \in C^{1}(\mathcal{N})$. On the set $U=\mathcal{N} \cap\{\rho \geq 0\}$ we have $\bar{\partial}(T f)=0$.

Proof. Observe that in the definition of $T$ the integration is just over $\partial M$. By (2.3)(2.6), we see that $T f$ is well-defined for $z$ in an open set $\mathcal{N}$, depending on $\rho$ only, containing $M$ in $\{\sigma<0\} \times \mathbb{C}^{n}$ and is $C^{k-2}$ there.

We show that $\bar{\partial}(T f)=0$ in the interior of $U$. Consider $m>1$ first. The identity (2.2) and type considerations imply $\bar{\partial}_{z} \Omega\left(\mathfrak{r}^{*}, \mathfrak{s}\right)=-\bar{\partial}_{\zeta} \Omega\left(\mathfrak{r}^{*}, \mathfrak{s}\right)$ in this case. Thus

$$
\begin{aligned}
\bar{\partial}(T f) & =(-1)^{n-1} \int_{\partial M} f \wedge \bar{\partial}_{z} \Omega\left(\mathfrak{r}^{*}, \mathfrak{s}\right)=(-1)^{n} \int_{\partial M} f \wedge \bar{\partial}_{\zeta} \Omega\left(\mathfrak{r}^{*}, \mathfrak{s}\right) \\
& =-\int_{\partial M} \bar{\partial}_{\zeta}\left(f \wedge \Omega\left(\mathfrak{r}^{*}, \mathfrak{s}\right)\right)=-\int_{\partial M} d_{\zeta}\left(f \wedge \Omega\left(\mathfrak{r}^{*}, \mathfrak{s}\right)\right)=0
\end{aligned}
$$

by Stokes' theorem. For $m=1$, the section $\mathfrak{s}$ is the Cauchy kernel which is holomorphic in both $\zeta$ and $z$. Hence (2.2) and type consideration give

$$
\bar{\partial}_{z}(T f)=(-1)^{n-1} \int_{\partial M} f \wedge \Omega\left(\mathfrak{r}^{*}\right) .
$$

For $z \in U \backslash M$ we can apply Stokes' theorem to the above integral and get $\bar{\partial}_{z}(T f)=$ 0 as $\bar{\partial} f=\bar{\partial}_{\zeta} \mathfrak{r}^{*}=0$

Since $T f \in C^{k-2}(\mathcal{N})$, we conclude that $\bar{\partial}(T f)=0$ on $U$ by continuity. The lemma is proved.

\section{Remark 3.3.}

(a) In (2.7) the form inside the parenthesis after $\bar{\partial}_{M}$ is in $C^{1}(M)$ provided that $f$ is in $C^{1}(M)$, and so it can be extended to a $C^{1}$ form in $\{\sigma<0\} \times \mathbb{C}^{n}$. By Lemma 3.2 the last form in (2.7) is actually in $C^{k-2}(U)$ and is $\bar{\partial}$-closed. Therefore, in this case, (2.7) becomes a "jump formula" for $f$. A more general jump formula can be found in [2], but we don't need it here. 
(b) In view of (2.10),(2.11) and Lemma 3.2, we see that all the coefficients of $T f$ are holomorphic in $z^{\prime}$.

Let $\tilde{\sigma}$ be a $C^{1}$ function defined in a neighborhood of $\{\sigma \leq 0\}$ such that $\{\tilde{\sigma}<0\} \subset$ $\{\sigma<0\}$. Set $\tilde{M}=\{\rho=0\} \cap\left(\{\tilde{\sigma}<0\} \times \mathbb{C}^{n}\right)$. Suppose $d \rho \wedge d \tilde{\sigma} \neq 0$ on $\partial \tilde{M}$. Denote by $b^{\prime}=(\tilde{b}^{\prime}, \underbrace{0, \cdots, 0}_{n})$, where $\tilde{b}^{\prime}$ is the section for the Bochner-Martinelli kernel in $\mathbb{C}^{m}$. For $f$ in the domain of $T$, define

$$
T^{\prime} f(z)=(-1)^{n-1} \int_{\partial \tilde{M}} f \wedge \Omega\left(\mathfrak{r}^{*}, b^{\prime}\right)(\zeta, z) .
$$

Lemma 3.1 and the proof of Lemma 3.2 still hold for $T^{\prime}$ and consequently $T^{\prime} f$ is a $\bar{\partial}$-closed form in $\tilde{U}=\mathcal{N} \cap\{\rho \geq 0\} \cap\left(\{\tilde{\sigma}<0\} \times \mathbb{C}^{n}\right)$. The next lemma shows that $T$ is "locally" defined over $\mathbb{C}^{m}$.

Lemma 3.4. Let $f$ be in the domain of $T$. Then $T f=T^{\prime} f$ on $\tilde{U}$.

Proof. For $z \in \tilde{U}$, apply (2.2) to get

$$
\begin{aligned}
T f= & (-1)^{n-1} \int_{\partial M} f \wedge \Omega\left(\mathfrak{r}^{*}, \mathfrak{s}\right)(\zeta, z) \\
= & (-1)^{n-1} \int_{\partial M} f \wedge\left(\Omega\left(\mathfrak{r}^{*}, b^{\prime}\right)-\Omega\left(\mathfrak{s}, b^{\prime}\right)-\bar{\partial}_{\zeta, z} \Omega\left(\mathfrak{r}^{*}, \mathfrak{s}, b^{\prime}\right)\right) \\
= & (-1)^{n-1} \int_{\partial M} f \wedge \Omega\left(\mathfrak{r}^{*}, b^{\prime}\right) \text { by Stokes' theorem and type considerations, } \\
= & (-1)^{n-1} \int_{\partial \tilde{M}} f \wedge \Omega\left(\mathfrak{r}^{*}, b^{\prime}\right) \\
& +(-1)^{n-1} \int_{M \backslash \tilde{M}} d_{\zeta}\left(f \wedge \Omega\left(\mathfrak{r}^{*}, b^{\prime}\right)\right) \text { by Stokes' theorem. }
\end{aligned}
$$

In the last integral we use (2.2) again to get

$$
d_{\zeta} \Omega\left(\mathfrak{r}^{*}, b^{\prime}\right)=\bar{\partial}_{\zeta} \Omega\left(\mathfrak{r}^{*}, b^{\prime}\right)=-\bar{\partial}_{z} \Omega\left(\mathfrak{r}^{*}, b^{\prime}\right)+\Omega\left(\mathfrak{r}^{*}\right)-\Omega\left(b^{\prime}\right)
$$

and the integral vanishes by type considerations. So

$$
T f=(-1)^{n-1} \int_{\partial \tilde{M}} f \wedge \Omega\left(\mathfrak{r}^{*}, b^{\prime}\right)=T^{\prime} f .
$$

This completes the proof. 
Lemma 3.4 has two implications. First, in Lemma 3.1 the assumption on $u$ can be weakened by $u \in C_{(0, n-2)}^{0}(M)$. Next, it suggests that the strong pseudoconvexity of the base set can be relaxed when one defines $T f$. This is seen more precisely in Lemma 3.5.

For $t \geq 0$, set

$$
\begin{aligned}
M_{t} & =\{\rho=t\} \cap\left(\{\sigma<0\} \times \mathbb{C}^{n}\right) \\
\tilde{M}_{t} & =\{\rho=t\} \cap\left(\{\tilde{\sigma}<0\} \times \mathbb{C}^{n}\right) \quad \text { and } \\
\Gamma_{z^{\prime}, t} & =\{\rho=t\} \cap\left(\left\{z^{\prime}\right\} \times \mathbb{C}^{n}\right) \quad \text { for } z^{\prime} \in\{\sigma<0\} .
\end{aligned}
$$

When $t=0$ we have $\Gamma_{z^{\prime}}=\Gamma_{z^{\prime}, 0}, \tilde{M}=\tilde{M}_{0}$, and $M=M_{0}$.

Now fix $z_{0}^{\prime} \in\{\sigma<0\}$. As in Lemma 3.4, let $\tilde{\sigma}=\left|z^{\prime}-z_{0}^{\prime}\right|^{2}-\epsilon^{2}$, where $\epsilon>0$ is chosen so that $\{\tilde{\sigma}<0\} \subset\{\sigma<0\}$ and $d \rho \wedge d \tilde{\sigma} \neq 0$ on $\partial \tilde{M}$. For $\epsilon$ small enough there exist $t>0$ small such that $\Gamma_{z_{0}^{\prime}, t}$ is strongly pseudoconvex in $\left\{z_{0}^{\prime}\right\} \times \mathbb{C}^{n}$ and

$$
\left\{z^{\prime}|| z^{\prime}-z_{0}^{\prime} \mid<\epsilon\right\} \times\left\{z^{\prime \prime} \mid\left(z_{0}^{\prime}, z^{\prime \prime}\right) \in \Gamma_{z_{0}^{\prime}, t}\right\} \subset \tilde{U} \backslash M .
$$

Fix such $\epsilon$ and $t$. By Lemma 3.4 we have for $z_{0} \in \tilde{U}$ satisfying $\rho\left(z_{0}\right)>t$

$$
\begin{aligned}
T f\left(z_{0}\right) & =(-1)^{n-1} \int_{\partial \tilde{M}} f(\zeta) \wedge \Omega\left(\mathfrak{r}^{*}, b^{\prime}\right)\left(\zeta, z_{0}\right) \\
& =(-1)^{n-1} \int_{\partial \tilde{M}}(T f)(\zeta) \wedge \Omega\left(\mathfrak{r}^{*}, b^{\prime}\right)\left(\zeta, z_{0}\right)
\end{aligned}
$$

where the last equality follows from (2.7) and Lemma 3.1 for $T^{\prime}$.

Since in the last integral

$$
\Omega\left(\mathfrak{r}^{*}, b^{\prime}\right)=R(\zeta, z) \wedge \Omega_{0}\left(b^{\prime}\right)
$$

where $R(\zeta, z)$ is a form holomorphic in $\zeta$. Applying Stokes' theorem to the last integral in the above formula, we have

$$
T f\left(z_{0}\right)=(-1)^{n-1} \int_{S_{\epsilon}}(T f)(\zeta) \wedge \Omega\left(\mathfrak{r}^{*}, b^{\prime}\right)\left(\zeta, z_{0}\right)
$$

by type consideration and the fact that $\bar{\partial}_{\zeta} \Omega_{0}\left(b^{\prime}\right)=0$, where $S_{\epsilon}=\left\{\zeta^{\prime}|| \zeta^{\prime}-z_{0}^{\prime} \mid=\right.$ $\epsilon\} \times\left\{\zeta^{\prime \prime} \mid\left(z_{0}^{\prime}, \zeta^{\prime \prime}\right) \in \Gamma_{z_{0}^{\prime}, t}\right\}$

Let $\epsilon$ tend to zero. It follows from Lemma 1.14 of [Ky] that

$$
T f\left(z_{0}\right)=(-1)^{n-1} c_{m} \int_{\zeta^{\prime \prime} \in \Gamma_{z_{0}^{\prime}, t}^{\prime}}(T f)\left(z_{0}^{\prime}, \zeta^{\prime \prime}\right) \wedge \Omega\left(\tilde{r}_{z_{0}^{\prime}, t}^{*}\right)\left(\zeta^{\prime \prime}, z_{0}^{\prime \prime}\right)
$$

where $c_{m}=\frac{(2 \pi i)^{m}}{(m-1) !}$ and $\tilde{\mathfrak{r}}_{z_{0}^{\prime}, t}^{*}$ is the section constructed from $\tilde{\rho}\left(z^{\prime \prime}\right)=\rho\left(z_{0}^{\prime}, z^{\prime \prime}\right)-t$. We thus have the following lemma: 
Lemma 3.5. For any $z_{0}^{\prime} \in\{\sigma<0\}$, for any $z_{0}=\left(z_{0}^{\prime}, z_{0}^{\prime \prime}\right) \in \tilde{U}$ and for any $t>0$ such that $\rho\left(z_{0}\right)>t$ and $\left\{z^{\prime \prime} \mid \rho\left(z_{0}^{\prime}, z^{\prime \prime}\right)<t\right\}$ is strictly pseudoconvex in $\tilde{U} \cap\left(\left\{z_{0}^{\prime}\right\} \times\right.$ $\mathbb{C}^{n}$ ), we have

$$
T f\left(z_{0}\right)=(-1)^{n-1} c_{m} \int_{\zeta^{\prime \prime} \in \Gamma_{z_{0}^{\prime}, t}}(T f)\left(z_{0}^{\prime}, \zeta^{\prime \prime}\right) \wedge \Omega\left(\tilde{\mathfrak{r}}_{z_{0}^{\prime}, t}^{*}\right)\left(\zeta^{\prime \prime}, z_{0}^{\prime \prime}\right) .
$$

In particular, if $\left\{z^{\prime \prime} \mid \rho\left(z_{0}^{\prime}, z^{\prime \prime}\right)<0\right\}$ is strictly pseudoconvex which holds for $z_{0}^{\prime}$ in a dense open set in $\{\sigma<0\}$, we have

$$
T f\left(z_{0}\right)=(-1)^{n-1} c_{m} \int_{\zeta^{\prime \prime} \in \Gamma_{z_{0}^{\prime}}} f\left(z_{0}^{\prime}, \zeta^{\prime \prime}\right) \wedge \Omega\left(\tilde{\mathfrak{r}}_{z_{0}^{\prime}}^{*}\right)\left(\zeta^{\prime \prime}, z_{0}^{\prime \prime}\right) .
$$

Proof. It remains to prove $\left(3 \cdot 1^{\prime}\right)$. The denseness of such $z_{0}^{\prime}$ follows from Sard's theorem. In view of the fact that (3.1) holds for any $0<t<\rho\left(z_{0}\right)$ with $\left\{z^{\prime \prime} \mid \rho\left(z_{0}^{\prime}, z^{\prime \prime}\right)<\right.$ $t\}$ strictly pseudoconvex in $\tilde{U} \cap\left(\left\{z_{0}^{\prime}\right\} \times \mathbb{C}^{n}\right),\left(3.1^{\prime}\right)$ is obtained by taking limit as such $t$ goes to 0 in (3.1) and by (2.7) and Stokes'theorem.

Remark 3.6. Formula (3.1) (or $\left(\left(3.1^{\prime}\right)\right)$ ) is of fundamental importance. It shows that $T f$ can be defined by $\rho$ only: the connectivity and the strong pseudoconvexity of the base set $\{\sigma<0\}$ can be relaxed. Moreover, it shows that $T f$ has a continuous extension to the set $U_{1}=\mathcal{N} \cap\{\rho \geq 0\} \cap\left(\{\sigma \leq 0\} \times \mathbb{C}^{n}\right)$.

Now instead of $\{\sigma<0\}$ we take the base set to be an arbitrary open set $B$ in $\mathbb{C}^{m}$. It is easy to see that all the preceding results about $T f$ still holds. Indeed, through (3.1) and $\left(\left(3.1^{\prime}\right)\right)$ the properties of $T f$ become even more transparent.

\section{The moment operator $\mathcal{M}_{h}(g)$ and the proof of Theorem $\mathbf{1 . 2}$}

Let $\rho$ be the same as before and $B$ be an arbitrary relatively compact open subset in $\mathbb{C}^{m}$. Set

$$
\tilde{M}=\{\rho=0\} \cap\left(B \times \mathbb{C}^{n}\right) .
$$

For an open neighborhood $O$ of $\tilde{M}$, we set

$$
\tilde{U}=O \cap\{\rho \geq 0\} \cap\left(B \times \mathbb{C}^{n}\right) .
$$

Let $g$ be a $C^{1} \bar{\partial}$-closed $(0, n-1)$ form in $\tilde{U}$. Let $V$ be an open set in $B$ and $h$ be a function holomorphic in a neighborhood of $\{\rho=0\} \cap\left(V \times \mathbb{C}^{n}\right)$. Define the moment operator of $g$ with respect to $h$ on $V$ by

$$
\mathcal{M}_{h}(g)\left(z^{\prime}\right)=\int_{\zeta^{\prime \prime} \in \Gamma_{z^{\prime}}} h\left(z^{\prime}, \zeta^{\prime \prime}\right) g\left(z^{\prime}, \zeta^{\prime \prime}\right) d \zeta^{\prime \prime}, \text { where } d \zeta^{\prime \prime}=d \zeta_{m+1} \wedge \cdots \wedge d \zeta_{m+n}
$$

Lemma 4.1. $\mathcal{M}_{h}(g)\left(z^{\prime}\right)$ is a holomorphic function in $V$. 
Proof. First observe that

$$
\mathcal{M}_{h}(g)\left(z^{\prime}\right)=\int_{\zeta^{\prime \prime} \in \Gamma_{z^{\prime}}} h\left(z^{\prime}, \zeta^{\prime \prime}\right) g_{1}\left(z^{\prime}, \zeta^{\prime \prime}\right) d \zeta^{\prime \prime}
$$

where $g_{1}$ is defined by (2.9). Fix $z_{0}^{\prime} \in V$. Choose a domain $D$ in $\mathbb{C}^{n}$ with $C^{1}$ boundary and a neighborhood $W$ of $z_{0}^{\prime}$ in $V$ such that

$$
\Gamma_{z_{0}^{\prime}} \subset\left\{z_{0}^{\prime}\right\} \times D, \quad\{\rho \leq 0\} \cap\left(W \times \mathbb{C}^{n}\right) \subset W \times D \quad \text { and } W \times \partial D \subset \tilde{U} .
$$

For any function $h$ holomorphic in $\overline{W \times D}$ it follows from Stokes' theorem and (2.9), (2.10) that

$$
\mathcal{M}_{h}(g)\left(z^{\prime}\right)=\int_{\Gamma_{z^{\prime}}} h\left(z^{\prime}, \zeta^{\prime \prime}\right) g_{1}\left(z^{\prime}, \zeta^{\prime \prime}\right) d \zeta^{\prime \prime}=\int_{\partial D} h\left(z^{\prime}, \zeta^{\prime \prime}\right) g_{1}\left(z^{\prime}, \zeta^{\prime \prime}\right) d \zeta^{\prime \prime}
$$

whenever $z^{\prime} \in W$. Now by (2.10)

$$
\bar{\partial}_{z^{\prime}} \mathcal{M}_{h}(g)\left(z^{\prime}\right)=\int_{\partial D} h\left(z^{\prime}, \zeta^{\prime \prime}\right) \bar{\partial}_{z^{\prime}} g_{1}\left(z^{\prime}, \zeta^{\prime \prime}\right) d \zeta^{\prime \prime}=-\int_{\partial D} h\left(z^{\prime}, \zeta^{\prime \prime}\right) \bar{\partial}_{\zeta^{\prime \prime}} g_{2}\left(z^{\prime}, \zeta^{\prime \prime}\right) d \zeta^{\prime \prime}
$$

whenever $z^{\prime} \in W$ and so

$$
\bar{\partial} \mathcal{M}_{h}(g)\left(z^{\prime}\right)=-\int_{\partial D} d_{\zeta^{\prime \prime}}\left(h\left(z^{\prime}, \zeta^{\prime \prime}\right) g_{2}\left(z^{\prime}, \zeta^{\prime \prime}\right)\right) d \zeta^{\prime \prime}=0 .
$$

This completes the proof.

Remark 4.2. Suppose $f$ is a $\bar{\partial}$-closed $(0, n-1)$ form in $C^{0}(\overline{\tilde{M}})$. In view of Remark 3.6, $T f$ is well-defined in $\tilde{U}=\mathcal{N} \cap\{\rho \geq 0\} \cap\left(B \times \mathbb{C}^{n}\right)$ where $\mathcal{N}$ is given by Lemma 3.2. Locally, for each $z^{\prime} \in B$ there is a small open neighborhood $W$ of $z^{\prime}$ contained in $B$ such that for $z \in \tilde{U} \cap\left(W \times \mathbb{C}^{n}\right) f$ can be represented as in (2.7), we see immediately that $\mathcal{M}_{h}(f)=\mathcal{M}_{h}(T f)$ for any holomorphic function $h$. In other words, the moment of $f$ is well-defined and is holomorphic in $z^{\prime}$.

With Remark 4.2 we have:

Corollary 4.3. Fix $z^{\prime} \in$ B. All the holomorphic moments of $f$ on $\Gamma_{z^{\prime}}$ vanish is equivalent to $T f(z)=0$ on $\tilde{U} \cap\left(\left\{z^{\prime}\right\} \times \mathbb{C}^{n}\right)$. Moreover, suppose $\Gamma_{z^{\prime}}$ is strictly pseudoconvex, equation $\bar{\partial}_{\Gamma_{z^{\prime}}} u=f$ is solvable on $\Gamma_{z^{\prime}}$ if and only if $T f(z)=0$ for all $z \in \tilde{U} \cap\left(\left\{z^{\prime}\right\} \times \mathbb{C}^{n}\right)$.

Proof. To prove the first statement, we need only show that $M_{h}(f)\left(z^{\prime}\right)=0$ for all $h$ holomorphic near $\Gamma_{z^{\prime}}$ implies $T f(z)=0$ on $\tilde{U} \cap\left(\left\{z^{\prime}\right\} \times \mathbb{C}^{n}\right)$. The proof of Lemma 4.1 and Remark 4.2 give

$$
\begin{aligned}
0 & =\mathcal{M}_{h}(f)\left(z^{\prime}\right)=\int_{\Gamma_{z^{\prime}}} h\left(z^{\prime}, \zeta^{\prime \prime}\right)(T f)_{1}\left(z^{\prime}, \zeta^{\prime \prime}\right) d \zeta^{\prime \prime} \\
& =\int_{\Gamma_{z^{\prime}, t}} h\left(z^{\prime}, \zeta^{\prime \prime}\right) T f\left(z^{\prime}, \zeta^{\prime \prime}\right) d \zeta^{\prime \prime}, \quad t>0
\end{aligned}
$$


whenever $\Gamma_{z^{\prime}, t} \subset \tilde{U}$ and $h$ is holomorphic near $\Gamma_{z^{\prime}, t}$. The last equality follows from (2.10), (2.11) and Stokes' theorem. Now suppose $\Gamma_{z^{\prime}, t}$ is strictly pseudoconvex. Let $h\left(z^{\prime}, \zeta^{\prime \prime}\right) d \zeta^{\prime \prime}=\Omega\left(\tilde{\mathfrak{r}}_{z^{\prime}}^{*}\right)$. By Lemma 3.5 we have $T f(z)=0$ for $z \in \tilde{U} \cap$ $\left(\left\{z^{\prime}\right\} \times \mathbb{C}^{n}\right), \rho(z) \geq t$. By Sard's theorem 0 is a limit point of such $t$ so the first statement is proved. The second statement follows from the first statement and Remark 1.3(a).

Theorem 4.4. Let $\rho$ be as in Theorem 1.2. Let B be a connected relatively compact open subset in $\mathbb{C}^{m}$. Set

$$
\tilde{M}=\{\rho=0\} \cap\left(B \times \mathbb{C}^{n}\right) .
$$

Let $f$ be a $(0, n-1)$ form in $C^{0}(\overline{\tilde{M}})$ with $\bar{\partial} f=0$ on $\tilde{M}$ in distribution sense. Let $A$ be a subset of $B$ not contained in any subvariety of codimension one in $B$. If for every $z^{\prime} \in A$ all the holomorphic moments of $f$ on $\Gamma_{z^{\prime}}$ vanish, then $T f$ vanishes identically on $\tilde{U}=\mathcal{N} \cap\{\rho \geq 0\} \cap\left(B \times \mathbb{C}^{n}\right)$ where $\mathcal{N}$ is defined in Lemma 3.2.

Proof. Step 1. The assumption on the set $A$ implies that there is a point $p \in \bar{B}$ such that the intersection of $A$ with any neighborhood of $p$ is not contained in a subvariety of codimension one in $B$. Let $V$ be any connected neighborhood of $p$ in $\bar{B}$. Let $h$ be any function holomorphic near $\tilde{M} \cap\left(V \times \mathbb{C}^{n}\right)$. By Lemma 4.1 $\mathcal{M}_{h}(f)\left(z^{\prime}\right)$ is a holomorphic function in $V \cap B$. Remark 4.2 and the assumption give that $\mathcal{M}_{h}(f)\left(z^{\prime}\right)=\mathcal{M}_{h}(T f)\left(z^{\prime}\right)=0$ for all $z^{\prime} \in V \cap A$. By the choice of $p$, we must have $\mathcal{M}_{h}(f)\left(z^{\prime}\right)$ identically equal to zero on $V$.

Step 2. Claim: There is a neighborhood $W$ of $p$ in $\bar{B}$ such that $T f$ vanishes identically on $\tilde{U} \cap\left(W \times \mathbb{C}^{n}\right)$.

Proof of the Claim. By Corollary 4.3 it suffices to show that for every $z^{\prime} \in W$, $\mathcal{M}_{h}(f)\left(z^{\prime}\right)=0$ for all $h$ holomorphic near $\Gamma_{z^{\prime}}$. If there is no such neighborhood, then there exists a sequence $\left\{z_{j}^{\prime}\right\}_{1}^{\infty} \subset B$ such that $z_{j}^{\prime} \rightarrow p$ as $j \rightarrow \infty$ and $\operatorname{Tf}\left(z_{j}^{\prime}, \cdot\right)$ does not vanish identically for all $j=1,2, \ldots$.

Choose $t>0$ so that $\Gamma_{p, t}$ is a $C^{k}$ strictly pseudoconvex real hypersuface in $\tilde{U} \cap\left(\{p\} \times \mathbb{C}^{n}\right)$. Let $W$ be a connected open neighborhood of $p$ in $B$ such that $W$ $\times\left\{z^{\prime \prime} \mid\left(p, z^{\prime \prime}\right) \in \Gamma_{p, t}\right\} \subset \tilde{U} \backslash \tilde{M}$.

For every $j=1,2, \ldots$ there is a function $h_{j}$ holomorphic near $\Gamma_{z_{j}^{\prime}}$ such that $\mathcal{M}_{h_{j}}(f)\left(z_{j}^{\prime}\right) \neq 0$. For simplicity we may assume that $\Gamma_{z_{j}^{\prime}}$ is strictly pseudoconvex in $\left\{z_{j}^{\prime}\right\} \times \mathbb{C}^{n}$ (or we do as in the proof of Corollary 4.3).

Fix $j$ so that $z_{j}^{\prime} \in W$. Set $D_{1}=\left\{z^{\prime \prime} \mid \rho\left(p, z^{\prime \prime}\right)<t\right\}$ and $D_{2}=\left\{z^{\prime \prime} \mid \rho\left(z_{j}^{\prime}, z^{\prime \prime}\right)<\right.$ 0 . By our choice both $D_{1}, D_{2}$ are strictly pseudoconvex domains in $\mathbb{C}^{n}$ and $D_{2} \Subset D_{1}$ for every $j=1,2, \ldots$ Since $\rho\left(z_{j}^{\prime}, \cdot\right)$ is plurisubharmonic in $D_{1}$ if $\rho$ is plurisubharmonic in $\tilde{U}$ and which can be assumed, it follows from Corollary 5.4.3 of [8] that $D_{1}, D_{2}$ form a Runge pair.

Thus $h_{j}\left(z_{j}^{\prime}, \cdot\right)$ can be uniformly approximated on $\bar{D}_{2}$ by functions holomorphic on $D_{1}$. Let $\left\{g_{k}\right\}_{k=1}^{\infty}$ be such a sequence of holomorphic functions on $D_{1}$. By Step 1 , 
for all $k \geq 1 \mathcal{M}_{g_{k}}(f)\left(z^{\prime}\right)=\mathcal{M}_{g_{k}}(T f)\left(z^{\prime}\right)=0$ for all $z^{\prime} \in W$. Therefore we must have $\mathcal{M}_{h_{j}}(f)\left(z_{j}^{\prime}\right)=0$, contradicting our assumption on $z_{j}^{\prime}$ and $h_{j}$. This completes the proof of the claim.

Step 3. Set $\mathcal{S} \equiv\left\{z^{\prime} \mid z^{\prime} \in B, T f\left(z^{\prime}, \cdot\right) \equiv 0\right.$ on $\left.\tilde{U} \cap\left(\left\{z^{\prime}\right\} \times \mathbb{C}^{n}\right)\right\}$. By Step $2 \mathcal{S}$ has non-empty interior. In fact, the argument in Step 2 shows that the interior of $\mathcal{S}$ is both open and closed in $B$. Since $B$ is connected, we conclude that $\mathcal{S}=B$. Theorem 4.4 is proved.

From the proof of Theorem 4.4 we have the following:

Corollary 4.5. Under the assumptions of Theorem 4.4, the following statements are equivalent:

(a) For all $z^{\prime} \in A$, every holomorphic moment of $f$ on $\Gamma_{z^{\prime}}$ vanishes.

(b) $T f(z) \equiv 0$ on $\tilde{U}$.

(c) $T f(z) \equiv 0$ for all $z \in \Gamma_{z^{\prime}}, z^{\prime} \in A$.

(d) $\bar{\partial}_{\Gamma_{z^{\prime}, t}} u=T f\left(z^{\prime}, \cdot\right)$ is solvable on $\Gamma_{z^{\prime}, t} \subset \tilde{U}$ for every $z^{\prime} \in A$ and for every $t \geq 0$, provided that $\Gamma_{z^{\prime}, t}$ is strictly pseudoconvex in $\left\{z^{\prime}\right\} \times \mathbb{C}^{n}$.

Corollary 4.6. Under the assumptions of Theorem 4.4, if the set $\left\{z^{\prime} \mid \Gamma_{z^{\prime}}=\emptyset, z^{\prime} \in\right.$ $B\}$ is not contained in a subvariety of codimension one in $B$, then $T f \equiv 0$ on $\tilde{M}$. In particular, if $B=\{\sigma<0\}$, then $\bar{\partial}_{M}$ is solvable at $q=n-1$.

On the other hand, we have

Remark 4.7. Let $M=\{\rho=0\} \cap\left(\{\tilde{\sigma}<0\} \times \mathbb{C}^{n}\right)$ where $\rho$ is as in the assumption of Theorem 4.4 and $\tilde{\sigma}$ is any $C^{1}$ function satisfying $d \rho \wedge d \tilde{\sigma} \neq 0$ on $\partial M$. Suppose $M$ and $\{\tilde{\sigma}<0\}$ are connected and $m<n$, then for $f \in C_{(0, q)}^{0}(\bar{M})$ satisfying $\bar{\partial}_{M} f=0$ in distribution sense on $M$ with $m \leq q \leq n+m-1$, considering type, the following representation formula holds for $z \in M$

$$
\begin{aligned}
(-1)^{q} f(z)= & \bar{\partial}\left\{\int_{M} f(\zeta) \wedge \Omega_{q-1}\left(\mathfrak{r}, \mathfrak{r}^{*}\right)(\zeta, z)+(-1)^{q} \int_{\partial M} f(\zeta) \wedge \Omega\left(\mathfrak{r}, \mathfrak{r}^{*}, b^{\prime}\right)(\zeta, z)\right\} \\
& +\int_{\partial M} f(\zeta) \wedge \Omega_{q}\left(\mathfrak{r}^{*}, b^{\prime}\right)(\zeta, z) .
\end{aligned}
$$

Note that the last integral vanishes for $m \leq q \leq n-2$ by type consideration. In other words, $\bar{\partial}_{M}$ is always solvable for $m \leq q \leq n-2$ in this case. Thus the tangential Cauchy-Riemann equation (*) is solvable at $q=n-1$ iff $T^{\prime} f(z)=T f(z)=0$. In view of Corollary 4.6, if $\{\tilde{\sigma}<0\}$ is not contained in $\pi(\{\rho=0\})$, the projection of $\{\rho=0\}$ to the $\mathbb{C}^{m}$ plane, then (*) is solvable at $q=n-1$.

Proof of Theorem 1.2. The solution $u$ is obtained from Corollary 4.5 and formula (2.7). The regularity of $u$ can be proved by routine procedure, see e.g. [14], and we omit the details here. 


\section{Proof of Theorem 1.1}

Let $P_{N}$ denote the unit polydisc in $\mathbb{C}^{N}$ as before. We are going to define a sequence of subdomains exhausting $\Omega$. First choose a sequence of $C^{\infty}$ real-valued strictly convex functions $\left\{\rho_{1, j}\right\}_{j=1}^{\infty}$ in $\mathbb{C}^{m+n}$ satisfying:

$\left(p_{1}\right)\left\{\rho_{1, j}<0\right\} \Subset\left\{\rho_{1, j+1}<0\right\} \Subset P_{m+n}$ for each $j \geq 1 ;$

$\left(p_{2}\right) \cup_{1}^{\infty}\left\{\rho_{1, j}<0\right\}=P_{m+n}$;

( $\left.p_{3}\right)$ for each $j=1,2, \ldots, \rho_{1, j}(z)=\rho_{1, j}\left(\left|z_{1}\right|, \ldots,\left|z_{m+n}\right|\right)$ and is symmetric in $\left|z_{k}\right|, k=1, \ldots, m+n$.

Next, for $j=1,2, \ldots$, set $C_{j}=\left\{z \in \mathbb{C}^{m+n}|| z_{k}\left|<1+\frac{1}{3 j}, k=1, \ldots, m,\right| z_{k} \mid<\right.$ $\left.\frac{1}{2}+\frac{1}{3 j}, k=m+1, \ldots, m+n\right\}$. Choose a sequence of $C^{\infty}$ real-valued strictly convex functions $\left\{\rho_{2, j}\right\}_{j=1}^{\infty}$ satisfying:

( $\left.p_{4}\right)\left\{\rho_{2, j}<0\right\} \Subset C_{j}$ for $j=1,2, \ldots$;

( $\left.p_{5}\right)\left\{\rho_{2, j}=0\right\} \subset C_{j} \backslash \bar{C}_{j+1}$;

( $p_{6}$ ) for each $j=1,2, \ldots, \rho_{2, j}(z)=\rho_{2, j}\left(\left|z_{1}\right|, \cdots,\left|z_{m+n}\right|\right)$ and is symmetric in $\left|z_{k}\right|, k=1, \ldots, m$ and is also symmetric in $\left|z_{k}\right|, k=m+1, \ldots, m+n$ respectively.

Define

$$
D_{j}=\left\{\rho_{1, j}<0\right\} \cap\left\{\rho_{2, j}>0\right\}, \text { for } j=1,2, \ldots .
$$

Clearly we have

$$
D_{j} \Subset D_{j+1}, \text { for } j=1,2, \ldots \text { and } \cup_{1}^{\infty} D_{j}=\Omega .
$$

Remark 5.1. Let $\pi$ be the orthogonal projection from $\mathbb{C}^{m+n}$ into $\mathbb{C}^{m}$. Set

$$
E_{j}=\left\{z \in \mathbb{C}^{m+n} \mid z^{\prime} \in \pi\left(D_{j}\right), \rho_{1, j}(z)<0\right\}, j=1,2, \ldots
$$

In addition to $\left(p_{1}\right)-\left(p_{6}\right)$, we choose $\rho_{1, j}, \rho_{2, j}$ so that $E_{j}$ is a relatively compact convex set in $\mathbb{C}^{m+n}$ for each $j=1,2, \ldots$. Such functions $\rho_{1, j}, \rho_{2, j}$ are easy to construct.

For each $j \geq 1$, the boundary of $D_{j}$ can be written as

$$
\partial D_{j}=\partial D_{j 1} \cup \partial D_{j 2},
$$

where $\partial D_{j 1}=\left\{\rho_{1, j}=0\right\} \cap\left\{\rho_{2, j} \geq 0\right\}$ and $\partial D_{j 2}=\left\{\rho_{2, j}=0\right\} \cap\left\{\rho_{1, j} \leq 0\right\}$. 


\section{Remark 5.2.}

(a) For each $j=1,2, \ldots$, we can choose strictly plurisubharmonic function $\sigma_{j} \in$ $C^{\infty}\left(\mathbb{C}^{m}\right)$ such that $\left\{\sigma_{j}<0\right\} \Subset P_{m}$ and $\partial D_{j 2} \Subset\left\{\sigma_{j}<0\right\} \times \mathbb{C}^{n}$. Set

$$
M_{j}=\left\{\rho_{2, j}=0\right\} \cap\left(\left\{\sigma_{j}<0\right\} \times \mathbb{C}^{n}\right), j=1,2, \ldots
$$

It follows from [1] that $\bar{\partial}_{M_{j}} u=g$ is solvable on $M_{j}$ for any $L^{p}, 1 \leq p \leq \infty$, $\bar{\partial}$-closed $(0, q)$ form $g$ on $M_{j}, 1 \leq q<n-1$. Furthermore, if $g \in C^{k}\left(\bar{M}_{j}\right)$ then $u \in C^{k}\left(M_{j}\right)$ for $j=1,2, \ldots$

(b) If the assumption of Theorem 1.1 is satisfied, then Corollary 4.5 implies that $T f \equiv 0$ on $\tilde{M}_{j}=\left\{\rho_{2, j}=0\right\} \cap\left(P_{m} \times \mathbb{C}^{n}\right)$ with $B=P_{m}$. Hence by Theorem 1.2 the conclusions in (a) for the solvability and regularity of $\bar{\partial}_{M_{j}} u=g$ on $M_{j}$ also hold for $q=n-1, j=1,2, \ldots$, provided that $\bar{\partial}_{M_{j}} g=0$ and $g \in C^{k}\left(\bar{M}_{j}\right)$, $k \geq 0$.

Lemma 5.3. Let $g$ be a $\bar{\partial}$-closed $C^{k}(0, q)$ form on $\Omega$ with $k$ any nonnegative integer and $1 \leq q<n-1$. For $j=1,2, \ldots$, the equation $\bar{\partial} v_{j}=g$ is solvable with $v_{j} \in C^{k}\left(D_{j}\right)$. In fact,

$$
\begin{aligned}
v_{j}= & -\int_{D_{j}} g \wedge \Omega(b)+\int_{\partial D_{j 1}} g \wedge \Omega\left(b, \mathfrak{r}_{1, j}\right)+\int_{\partial D_{j 2}} g \wedge \Omega\left(b, \mathfrak{r}_{2, j}^{*}\right) \\
& +(-1)^{q+1} \int_{\partial D_{j 1} \cap \partial D_{j 2}} g \wedge \Omega\left(b, \mathfrak{r}_{1, j}, \mathfrak{r}_{2, j}^{*}\right)-\int_{\partial D_{j 1} \cap \partial D_{j 2}} u_{j} \wedge \Omega\left(\mathfrak{r}_{1, j}, \mathfrak{r}_{2, j}^{*}\right)
\end{aligned}
$$

where $b$ is the Bochner-Martinelli section in $\mathbb{C}^{m+n} ; \mathfrak{r}_{1, j}, \mathfrak{r}_{2, j}$ are sections corresponding to $\rho_{1, j}, \rho_{2, j}$; and $u_{j}$ is the $C^{k}$ solution to $\bar{\partial}_{M_{j}} u_{j}=g$ on $M_{j}$ in view of Remark 5.2(a).

Proof. Since $\rho_{2, j}$ is a smooth strictly convex function in $\mathbb{C}^{m+n}$, the section $\mathfrak{r}_{2, j}^{*}(\zeta, z)$ is well-defined for all $z \in\left\{\rho_{2, j}>0\right\}$ as long as $\zeta \in\left\{\rho_{2, j} \leq 0\right\}$. As usual, one starts from the formula:

$$
g(z)=-\bar{\partial}\left(\int_{D_{j}} g \wedge \Omega(b)(\zeta, z)\right)+\int_{\partial D_{j}} g \wedge \Omega(b)(\zeta, z) .
$$

The lemma is proved by repeated use of (2.2), Stokes' theorem and type considerations when interploating the integrals with $\Omega\left(\mathfrak{r}_{1, j}\right), \Omega\left(\mathfrak{r}_{2, j}^{*}\right)$, etc.. We omit the routine computations.

By part (b) of Remark 5.2 we have:

Corollary 5.4. Let $g$ be a $C^{k}, k \geq 0, \bar{\partial}$-closed $(0, n-1)$ form on $\Omega$ satisfying the assumption of Theorem 1.1 Then $\bar{\partial}_{D_{j}} v_{j}=g$ is solvable for every $j \geq 1$ with $v_{j} \in C_{(0, n-2)}^{k}\left(D_{j}\right)$. 
Proof of Theorem 1.1. By Corollary 5.4 it remains to construct a $C^{k}$ solution $v$ on $\Omega$ out of $v_{j}, j=1,2, \ldots$. The process below is a modification of [11, Lemma 3] which deals with $k=\infty$.

Consider the case $n>2$ first. Let $v_{j}$ be given by Corollary 5.4. Set $\tilde{v}_{0}=v_{3}$. Obviously $v_{3}-v_{4}$ is a $\bar{\partial}$-closed form in $C_{(0, n-2)}^{k}\left(D_{3}\right)$. By Lemma 5.3 there exists $w_{1} \in C_{(0, n-3)}^{k}\left(D_{2}\right)$ so that $\bar{\partial} w_{1}=v_{3}-v_{4}$ in $D_{2}$. Let $\chi_{1} \in C_{0}^{\infty}\left(D_{2}\right)$ such that $\chi_{1} \equiv 1$ on $\bar{D}_{1}$. Set

$$
\tilde{v}_{1}=v_{4}+\bar{\partial}\left(\chi_{1} w_{1}\right) \in C_{(0, n-2)}^{k}\left(D_{4}\right) .
$$

Then $\tilde{v}_{1}=v_{3}=\tilde{v}_{0}$ on $D_{1}$ and $\bar{\partial} \tilde{v}_{1}=f$ on $D_{4}$. We use induction to construct $\tilde{v}_{j}$ for $j>1$. Suppose we already have $\tilde{v}_{j} \in C_{(0, n-2)}^{k}\left(D_{j+3}\right)$ with $\bar{\partial} \tilde{v}_{j}=f$ on $D_{j+3}$ and $\tilde{v}_{j}=\tilde{v}_{j-1}$ on $D_{j}$. Now $\tilde{v}_{j}-v_{j+4} \in C_{(0, n-2)}^{k}\left(D_{j+3}\right)$ and $\bar{\partial}\left(\tilde{v}_{j}-v_{j+4}\right)=0$ on $D_{j+3}$. By Lemma 5.3 there exists $w_{j+1} \in C_{(0, n-3)}^{k}\left(D_{j+2}\right)$ so that $\bar{\partial} w_{j+1}=\tilde{v}_{j}-v_{j+4}$ on $D_{j+2}$. Choose $\chi_{j+1} \in C_{0}^{\infty}\left(D_{j+2}\right)$ such that $\chi_{j+1} \equiv 1$ on $\bar{D}_{j+1}$. Set

$$
\tilde{v}_{j+1}=v_{j+4}+\bar{\partial}\left(\chi_{j+1} w_{j+1}\right) \in C_{(0, n-2)}^{k}\left(D_{j+4}\right) .
$$

We have $\tilde{v}_{j+1}=\tilde{v}_{j}$ on $D_{j+1}$ and $\bar{\partial} \tilde{v}_{j+1}=f$ on $D_{j+4}$. In this way we get $v=$ $\lim _{j \rightarrow \infty} \tilde{v}_{j}$ in $C_{(0, n-2)}^{k}(D)$ and $\bar{\partial} v=f$ on $D$. This proves Theorem 1.1 when $n>2$.

When $n=2$, let $E_{j}$ be as in Remark 5.1. As $n=2>1$, every function holomorphic in $D_{j}$ extends holomorphically to $E_{j}$ by Hartogs theorem. Since $E_{j}$ is a bounded convex set in $\mathbb{C}^{m+2}$; it is a Runge domain in $\mathbb{C}^{m+2}$ (see [7, Theorem 4.7.8]). So the assumption of [11, Lemm 3] is satisfied and the case $n=2$ is proved. This completes the proof of Theorem 1.1.

With Corollary 5.4 replaced by Lemma 5.3 in the proof of Theorem 1.1 we immediately have:

Corollary 5.5. Let $g$ be a $C^{k} \bar{\partial}$-closed $(0, q)$ form, $1 \leq q \leq n-2$, on $\Omega$, where $k$ is any non-negative integer. Then there exists a $C^{k}(0, q-1)$ form $u$ on $\Omega$ such that $\bar{\partial} u=g$.

Note that Corollary 5.5 is part of Frenkel's lemma if $k=\infty$. The case $n \leq q \leq$ $m+n$ will be proved elsewhere.

There are many applications of the proof of Theorem 1.1, for example we have:

Corollary 5.6. Let $D$ be any bounded pseudoconvex domain in $\mathbb{C}^{N}$. Let $k$ be any non-negative integer. For any $\bar{\partial}$-closed $C^{k}(0, q)$ form $g$ on $D, 1 \leq q \leq N$, there exists a $C^{k}(0, q-1)$ form $u$ on $D$ such that $\bar{\partial} u=g$.

Moreover, if $g \in L^{p}(D), 1 \leq p \leq \infty$, then there exists $u \in L_{l o c}^{p}(D)$ such that $\bar{\partial} u=g$. 
Corollary 5.7. Let $\rho$ be a $C^{k}$ real-valued function on $\mathbb{C}^{m+n}$ which is strictly plurisubharmonic near $\{\rho \leq 0\}, 3 \leq k \leq \infty$. Let $B$ be a relatively compact pseudoconvex domain in $\mathbb{C}^{m}$. Set $M=\{\rho=0\} \cap\left(B \times \mathbb{C}^{n}\right)$. Let $f$ be a $C^{k^{\prime}}$ $\bar{\partial}$-closed $(0, q)$ form on $M, 0 \leq k^{\prime} \leq k-3$. Then there exists $u \in C_{(0, q-1)}^{k^{\prime}}(M)$ such that $\bar{\partial} u=f$ on $M$ if $1<q<n-1$.

\section{References}

[1] C. H. ChANG and H. P. LeE, Semi-global solution of $\bar{\partial}_{b}$ with $L^{p}(1 \leq p \leq \infty)$ bounds on strongly pseudoconvex real hypersurfaces in $\mathbb{C}^{n}(n \geq 3)$, Publ. Mat. 43 (1999), 535-570.

[2] C. H. ChANG and H. P. LEE, Hartogs theorem for CR functions, Bull. Inst. Math. Acad. Sinica 32 (2004), 221-227.

[3] J. E. FornæeSS, Embedding strictly pseudoconvex domains in convex domains, Amer. J. Math. 98 (1976), 529-569.

[4] F. HARTOGS, Zur Theorie der analytischen Funktionen mehrerer unabhängiger Veränderlichen, insbesondere über die Darstellung derselben durch Reihen, welche nach Potenzen einer Veränderlichen fortschreiten, Math. Ann. 62 (1906), 1-88.

[5] R. HARVEY and J. PolKIng, Fundamental solutions in complex analysis, I, II, Duke Math. J. 46 (1979), 253-340.

[6] G. M. HENKIn, The Lewy equation and analysis on pseudoconvex manifolds, Russian Math. Surveys 32 (1977), 59-130.

[7] L. HÖRmANDER, "Notions of Convexity", Progress in Math., Vol. 127, Birkhäuser, 1994.

[8] S. G. KRAnTZ, "Function Theory of Several Complex Variables", 2ed. Wadsworth Books/Cole Mathematics Series, 1992.

[9] R. Narasimhan, "Several Complex Variables", Chicago Lecture Notes in Math., The University of Chicago Press, 1971.

[10] M. R. RANGE, "Holomorphic Functions and Integral Representations in Several Complex Variables", Springer-Verlag, New York, 1986.

[11] J. P. RosAY, Some application of Cauchy-Fantappié forms to (local) problems in $\bar{\partial}_{b}$, Ann. Scuola Norm. Sup. Pisa Cl. Sci. (4), 13 (1986), 225-243.

[12] Y. T. SIU, "Techniques of Extension of Analytic Objects", Lecture Notes in Pure and Applied Math., Vol. 8, Marcel Dekker, 1974.

[13] Y. T. Siu and G. Trautmann, "Gap-sheaves and Extension of Coherent Analytic Subsheaves", Lecture Notes in Mathematics, Vol. 172, Springer-Verlag, 1971.

[14] S. M. Webster, On the local solution of the tangential Cauchy-Riemann equations, Nonlinear Anal. 6 (1989), 167-182.

Institute of Mathematics

Academia Sinica

Taipei, Taiwan, R.O.C.

pino@math.sinica.edu.tw

hplee@math.sinica.edu.tw 